\title{
Type and occurrence of serious complications in patients after mild traumatic brain injury
}

\author{
Sivak $\mathrm{S}^{1}$, Nosal $\mathrm{V}^{1}$, Bittsansky $\mathrm{M}^{2}$, Dluha $\mathrm{J}^{1}$, Dobrota $\mathrm{D}^{2}$, Kurca $\mathrm{E}^{1}$ \\ Clinic of Neurology, Jessenius Medical Faculty, Comenius University, Martin, Slovakia. \\ egonkurca@gmail.com
}

\begin{abstract}
Traumatic brain injury (TBI) remains a major public health and socio-economic problem, and $70-90 \%$ of all TBIs are classified as mild. Mild TBIs and concussions are mostly considered to be non-serious conditions with symptoms subsiding within a few days or weeks. However in $10-15 \%$ of patients, the symptoms persist one year after concussion and mostly include headache, fatigue, irritability, and cognitive problems (e.g. memory, concentration). These persisting symptoms negatively influence patient daily activities as postconcussion syndrome (PCS). Second-impact syndrome (SIS) is a very rare but usually fatal condition and occurs when repeated brain injuries lead to a catastrophic diffuse brain swelling. There is no scientific evidence on the incidence and risk of SIS. Chronic traumatic encephalopathy (CTE) is a progressive degenerative disease of the brain found in patients with a history of repetitive brain trauma. CTE presents with behavioural, cognitive, and motor symptoms. The literature to date lacks prospective epidemiological studies of the incidence of CTE. In recent medical literature, there is a description of 110 athletes with postmortem diagnosis of CTE (Tab. 1, Ref. 37). Text in PDF www.elis.sk.

KEY WORDS: mild traumatic brain injury; concussion; postconcussion syndrome; second-impact syndrome; chronic traumatic encephalopathy.
\end{abstract}

\section{Mild traumatic brain injury}

Traumatic brain injury (TBI) remains a major public health and socio-economic problem. It is estimated that $70-90 \%$ of all TBIs are classified as mild (1). European Federation of Neurological Societies defines mild traumatic brain injury (MTBI) as a head injury with Glasgow Coma Scale (GCS) score of 13-15, and with loss of consciousness (if present) lasting 30 minutes or less (2). Concussion (commotio cerebri) is another traditional term still used in medical terminology in some European countries and it is widely used in sports medicine (3). Concussion is a specific kind of MTBI defined as posttraumatic transient impairment of neurological function that resolves spontaneously and is associated with normal standard structural neuroimaging findings (3). MTBIs and concussions are mostly considered to be non-serious conditions with symptoms subsiding within a few days or weeks.

However in some patients, symptoms persist as a postconcussion syndrome (PCS) and negatively influence their daily activities. Several cases of second-impact syndrome (SIS) have been

${ }^{1}$ Clinic of Neurology, Jessenius Medical Faculty, Comenius University, Martin, Slovakia, and ${ }^{2}$ Department of Medical Biochemistry, Jessenius Medical Faculty, Comenius University, Martin, Slovakia

Address for correspondence: E. Kurca, PhD, FESO, Clinic of Neurology, JLF UK, Kollarova 2, 03659 Martin, Slovakia.

Phone: +421.43.4203209, Fax: +421.43.4131005

Acknowledgement: This work was supported by project Center of excellence for research in personalized therapy (CEVYPET; ITMS 26220120053), which is co-financed from EC sources. described as a catastrophic complication with high mortality and morbidity in younger athletes after repeated MTBI. There is a growing number of case reports with progressive brain neurodegeneration called chronic traumatic encephalopathy (CTE) in patients after multiple concussions. In our paper, we would like to focus on these rare and serious complications after MTBI.

\section{Postconcussion syndrome}

Postconcussion syndrome (PCS) is defined by the 10th revision of International Classification of Diseases (ICD-10, dg. F07.2) as an occurrence of at least three symptom categories within one month of injury. The categories include somatic symptoms (headache, dizziness, malaise, fatigue, noise intolerance), emotional symptoms (irritability, depression, anxiety, lability), cognitive symptoms (concentration, memory, intellectual difficulties), insomnia, and reduced alcohol tolerance.

In our unpublished longitudinal case-control study, postconcussion syndrome was present in $62.5 \%$ of our patients $3-7$ months after MTBI. The most common symptoms were poor memory $(62.5 \%)$, irritability $(54.2 \%)$, headache $(54.2 \%)$, drowsiness (50\%), and concentration problems (45.8\%). These findings are consistent with results from other studies. In general population, the prevalence of PCS at 3 months post-injury is between 24 and $84 \%$ (4). It is estimated that $10-15 \%$ of individuals are reported to remain symptomatic one year after concussion (5). These symptoms are not specific for PCS and are associated with other clinical and non-clinical groups including healthy adults (in our 
study, $57.1 \%$ of controls reported symptoms consistent with PCS), and patients suffering from chronic pain, spinal injury, non-brain trauma, or psychological distress (6).

Whether PCS is experienced after MTBI appears to depend on different neurobiological and psychological factors, including pre-injury depression and anxiety, somatoform disorder, lifestressors, pain, female gender, older age, coping style, cognitive biases such as expectation of symptoms, premature return to full training regime in sport, litigation stress, exaggeration, and malingering $(6,7)$.

The differential diagnosis of PCS is a multidisciplinary problem and includes headache disorders, cervical injury, visual and vestibular dysfunction, psychiatric disorders such as anxiety, depression or somatization, and malingering.

A single psychoeducational session with support and education about the symptoms, expected recovery and gradual resumption of activities prevents or shortens the duration of postconcussion symptoms (8). There are potential benefits of structured and supervised exercise during the post-acute concussion recovery period (6). Lengthy physical inactivity in patients with chronic PCS can lead to prolonged recovery (9). Referral to various specialists should be made according to chronic somatic symptoms as well as mood, cognition, and sleep impairment. Psychoterapy, cognitivebehavioural therapy, education, cognitive training, physiotherapy, or vestibular rehabilitation may aid recovery. For refractory PCS, symptomatic pharmacologic treatment can be helpful (e.g. SSRI, analgesics; hypnotics) (10).

\section{Second-impact syndrome}

Second-impact syndrome (SIS) is a very rare condition and occurs when an athlete sustains an initial mild head injury, then suffers a second head injury before the symptoms associated with the first impact disappear. After the second impact, the athlete's condition quickly deteriorates and is often fatal (11). Most of those who survive are severely disabled (12). It was first described in 1973 by Richard Schneider in two athletes and the term secondimpact syndrome was coined in 1984 by Saunders and Harbaugh. SIS was described in young athletes, predominantly males (90\%) at age of 10 to 24 (mean 17.9 years). Among afflicted athletes were American football players (71\%), boxers (14\%), and occasionally martial artists, skiers, and ice-hockey players $(13,14)$. It is assumed, that SIS is caused by catastrophic cerebral oedema resulting from post-traumatic loss of cerebral blood-flow autoregulation combined with stress-induced catecholamine release with high blood pressure (15). Apart from diffuse cerebral oedema, a thin acute subdural hematoma has been described in some cases (16).

Typically, the athlete reports postconcussion symptoms after the first mild traumatic brain injury, such as headache, balance problems, attention and memory problems. Before these symptoms are fully cleared, which may take days or weeks (0-32 days), the patient suffers a second head injury. Cantu and Gean stated that the second injury may be remarkably minor or it can be only a blow to the chest that indirectly leads to transmission of impulsive force to the head and brain. The athlete may appear stunned after the injury, but usually does not experience loss of consciousness. Within several seconds or minutes, the athlete suddenly falls to the ground, is semicomatose or comatose with dilated pupils, and develops respiratory failure. Clinical deterioration occurs more rapidly than usually seen in cases of epidural hematoma (16).

The patient needs cardiopulmonar resuscitation and fast transport to emergency department. Computed tomography (CT) is the initial examination of choice and when intracranial hypertension is detected, neurosurgical intervention and oedema treatment are indicated (16).

This very rare condition still remains controversial. There is no scientific evidence on the incidence and risk of SIS, other than reported case-series from North America. It is not clear whether the initial concussion increases the risk for cerebral swelling after the second injury. It has been suggested that SIS and malignant cerebral oedema after one MTBI could be manifestations of the same pathology $(13,17,18,19)$. In the only systematic review of this topic, a total of 17 cases of reported SIS were identified in the world literature. Of these, only five cases actually suffered a repeated injury (20). Despite these controversies, all return-to-play guidelines recommend that athletes must not return to competition until all concussion symptoms are absent, both at rest and during exercise (3).

\section{Chronic traumatic encephalopathy}

It has long been known that multiple MTBIs result in late-time dementia. This was initially recognized by American pathologist Dr. Harrison S. Martland in 1928, who described a spectrum of persistent motor and neuropsychiatric symptoms in former professional boxers $(21,22)$. He coined this syndrome punch-drunk syndrome due to the early signs resembling intoxication, such as dominant unsteadiness, slowed-down movements, and mental confusion. Since 1928, further case reports and series of boxers have been described in medical literature, indicating that repetitive brain injuries can induce chronic and potentially progressive neuropsychiatric symptoms on a neuropathological basis (23). The syndrome was termed with several names such as traumatic encephalopathy of professional pugilists (24), dementia pugilistica (25), chronic progressive traumatic encephalopathy of boxers (26), and chronic traumatic encephalopathy (CTE) (27). The first detailed neuropathological findings of a patient with CTE were described by Brandenburg and Hallervorden in 1954 and later in a group of 15 boxers by Corsellis (28). In 2005, Omal et al described for the first time a case report of an American football player with progressive neuropsychiatric disorder with neuropathological findings similar to those found in boxers with punch-drunk syndrome (29). The understanding that the disorder was not restricted to boxing (pugilism), the broader term of chronic traumatic encephalopathy (CTE) has began to be favoured. CTE has been described in several sports such as American football, ice hockey, wrestling (30), and rugby (13), but also in non-sport cases such as war veterans (31), epilepsy, physical violence (32), self-injury (33), and alcoholics with history of repeated falls (34). At the moment, there is a cohort of 110 athletes with postmortem diagnosis of CTE (23). 
Tab. 1. Clinical presentation of CTE (32).

\begin{tabular}{l}
\hline Behavioural and psychiatric features \\
\hline Aggression and/or agitation \\
Apathy \\
Impulsivity \\
Depression \\
Delusions (such as paranoia) \\
Suicidal behaviour \\
Cognitive features \\
\hline Impaired attention and concentration \\
Memory problems \\
Executive dysfunction \\
Dementia \\
Visuospatial difficulties \\
Language impairment \\
Motor features \\
\hline Dysarthria, including scanning speech \\
Spasticity \\
Ataxia, including incoordination \\
Parkinsonism, including tremors \\
Gait disturbance \\
Motor neuron disease (possibly)
\end{tabular}

The literature to date lacks prospective epidemiological studies of the incidence of CTE (23).

CTE presents with behavioural, cognitive, and motor symptoms (Tab. 1). The typical onset is between 30 and 65 years of age (35). When compared to other neurodegenerative diseases such as Alzheimer's disease and frontotemporal lobar degeneration, CTE progression tends to be slower (in tens of years) (36). The earliest findings in patients with CTE are mostly behavioural in form of mood changes including poor impulsivity control, aggression, explosive behaviour, risky behaviour leading to bankruptcy, substance abuse, apathy, and depression with high incidence of suicide. Among cognitive changes the early ones are impaired attention, executive dysfunction, and memory problems. Motor symptoms tend to occur in later stages of the disease (35).

Pathological findings in CTE differ from other neurodegenerative diseases/tauopathies such as Alzheimer's disease and frontotemporal lobar degeneration (37). Typical macroscopic pathological findings in patients with CTE are brain atrophy, enlargement of ventricles, cavum septum pellucidum and pallor of the locus coeruleus and substantia nigra. Typical microscopic findings are neurofibrillary tangles (in 95-100\% of patients), TDP-43 inclusions $(86 \%)$, neuritic amyloid $\beta$ plaques $(30-53 \%)(30,36)$. In the largest study of patients with CTE to date, McKee et al classify CTE progression in four stages (36). Pathological process with findings of neurofibrilary tangles with phosphorylated tau protein begins focally, perivascularly in sulcular depths of frontal cortices (Stage 1) and spreads during the years up to most regions of the cortex, medial temporal structures, brainstem, and spinal cord (Stage 4). Due to these pathological findings it is suggested that CTE belongs to the group of progressive tauopathies $(32,36)$. We still do not know the exact pathophysiological mechanisms of tau protein hyperphosphorylation, oligomerization, and transfer from cell to cell. McKee et al have suggested that repeated head injuries and deposits of hyperphosphorylated tau protein lead to other protein deposits such as TDP-43, amyloid $\beta$ and $\alpha$-synuclein.
Clinical diagnosis of CTE can be problematic as the development of chronic progressive symptoms manifest typically after a period of latency (mostly several years) after the last brain injury. Jordan has proposed clinical criteria for CTE, which have not been validated yet (35). The definitive diagnosis requires neurological signs consistent with CTE and pathological confirmation. The probable diagnosis needs two or more of the following conditions: 1) cognitive and/or behavioral impairment; 2) cerebellar dysfunction; and 3) pyramidal tract disease or extrapyramidal disease. It has to be consistent with clinical description of CTE and clinically distinguishable from other disease processes. The possible CTE must be consistent with clinical description of CTE, but could be explained by other disease processes. The improbable CTE is inconsistent with clinical description of CTE and can be explained by other disease processes that are unrelated to brain trauma.

The differential diagnosis of CTE includes PCS and other neurodegenerative forms of dementia. Postconcussion syndrome has an acute onset and is temporally related to mild brain injury (concussion) without a latent period (35). If patients with PCS show signs of progression in neuropsychiatric symptoms several years after the last injury, they should be checked for the symptoms of CTE. The differential diagnosis of early CTE with respect to Alzheimer's disease is difficult. Brain trauma in personal history is a risk factor for both diagnoses. Patients with early stages with CTE tend to present with chronic headache and dominant behavioural and psychiatric symptoms (e.g. depression, mood swings, substance abuse, disinhibition). Dementia with short term memory deficits and parkinsonism are typical for later stages of CTE. In younger patients with repeated brain trauma, the diagnosis of CTE should be favoured. AD is infrequent in this younger population (22). Both, the behavioural variant of frontotemporal lobar degeneration (bvFLD) and CTE affect younger patients and both are associated with motor neuron disease. Unlike CTE, bvFLD has a faster progression and different memory impairment characteristics (37). When comparing CTE to dementia with Lewy bodies (DLB), the combination of dementia and parkinsonism tend to occur early in DLB but not in CTE. Mood swings with aggression and explosive behaviour are typical for CTE, but not for DLB (37).

There is no treatment for CTE but unlike other forms of dementia, it is preventable. More should be done in protection of players by changing the rules of sports, medical guidelines and modernising the protective equipment.

\section{References}

1. Cassidy JD, Carroll LJ, Peloso PM, Borg J, von Holst H, Holm L, Kraus J, Coronado VG; WHO Collaborating Centre Task Force on Mild Traumatic Brain Injury. Incidence, risk factors and prevention of mild traumatic brain injury: results of the WHO Collaborating Centre Task Force on Mild Traumatic Brain Injury. J Rehabil Med 2004; 43 (Suppl): 28-60.

2. Vos PE, Alekseenko Y, Battistin L, Ehler E, Gerstenbrand F, Muresanu DF, Potapov A, Stepan CA, Traubner P, Vecsei L, von Wild K; European Federation of Neurological Societies. Mild traumatic brain injury. Eur J Neurol 2012; 19 (2): 191-198. 
3. McCrory P, Meeuwisse WH, Aubry M, Cantu B, Dvorák J, Echemendia RJ, Engebretsen L, Johnston K, Kutcher JS, Raftery M, Sills A, Benson BW, Davis GA, Ellenbogen RG, Guskiewicz K, Herring SA, Iverson GL, Jordan BD, Kissick J, McCrea M, McIntosh AS, Maddocks D, Makdissi M, Purcell L, Putukian M, Schneider K, Tator CH, Turner M. Consensus statement on concussion in sport: the 4th International Conference on Concussion in Sport held in Zurich, November 2012. Br J Sports Med 2013; 47 (5): 250-258.

4. Ryan LM, Warden DL. Postconcussion syndrome. Int Rev Psychiatry 2003; 15 (4): 310-316.

5. Alexander MP. Mild traumatic brain injury: pathophysiology, natural history, and clinical management. Neurology 1995; 45: 1253-1260.

6. Broshek DK, De Marco AP, Freeman JR. A review of post-concussion syndrome and psychological factors associated with concussion. Brain Inj 2015; 29 (2): 228-237.

7. McCrea MA. Mild traumatic brain injury and post- concussion syndrome. New York: Oxford University Press, Inc. 2008.

8. Miller LJ, Mittenberg W. Brief cognitive behavioral interventions in mild traumatic brain injury. Appl Neuropsychol 1998; 5 (4): 172-183.

9. Silverberg ND, Iverson GL. Is rest after concussion "the best medicine?": recommendations for activity resumption following concussion in athletes, civilians, and military service members. J Head Trauma Rehabil 2013; 28 (4): 250-259.

10. Barlow KM. Postconcussion Syndrome: A Review. J Child Neurol 2014.

11. Cantu RC. Second impact syndrome: Immediate management. Phys Sportsmed 1995; 20: 55-58.

12. Potts MA, Stewart EW, Griesser MJ, Harris JD, Gelfius CD, Klamar K. Exceptional neurologic recovery in a teenage football player after second impact syndrome with a thin subdural hematoma. PM R 2012; 4 (7): 530-532.

13. McKee AC, Daneshvar DH, Alvarez VE, Stein TD. The neuropathology of sport. Acta Neuropathol 2014; 127 (1): 29-51.

14. Mori T, Katayama Y, Kawamata T. Acute hemispheric swelling associated with thin subdural hematomas: pathophysiology of repetitive head injury in sports. Acta Neurochir 2006; Suppl (96): 40-43.

15. Wetjen NM, Pichelmann MA, Atkinson JL. Second impact syndrome: concussion and second injury brain complications. J Am Coll Surg 2010; 211 (4): 553-557.

16. Cantu RC, Gean AD. Second-impact syndrome and a small subdural hematoma: an uncommon catastrophic result of repetitive head injury with a characteristic imaging appearance. J Neurotrauma 2010; 27 (9): $1557-1564$

17. Donovan J, Cancelliere C, Cassidy JD. Summary of the findings of the International Collaboration on Mild Traumatic Brain Injury Prognosis. Chiropr Man Ther 2014; 22 (1): 38.

18. De Riggo J, Kolarovszki B, Richterová R, Kolarovszká H, Šutovský J, Ďurdík P. Measurement of the blood flow velocity in the pericallosal artery of children with hydrocephalus by transcranial Doppler ultrasonography - preliminary results. Biomed Pap Med Fac Univ Palacky Olomouc Czech Repub 2007; 151 (2): 285-289.
19. Kolarovszki B, De Riggo J. Hodnotenie klinických príznakov intrakraniálnej hypertenzie vo vzt’ahu k indikácii drenážneho výkonu u novorodencov a dojčiat s hydrocefalom. ČesSlov Pediat 2008; 63 (10): 521-527.

20. McCrory P. Does second impact syndrome exist? Clin J Sport Med 2001; 11 (3): 144-149.

21. Martland H. Punch drunk. JAMA 1928; 91: 1103-1107.

22. Shively S, Scher AI, Perl DP, Diaz-Arrastia R. Dementia resulting from traumatic brain injury: what is the pathology? Arch Neurol 2012; 69 (10): 1245-1251.

23. Solomon GS, Zuckerman SL. Chronic traumatic encephalopathy in professional sports: retrospective and prospective views. Brain Inj 2015; 29 (2): 164-170.

24. Parker HL. Traumatic encephalopathy ('punch drunk') of professional pugilists. J Neurol Psychopathol 1934; 15: 20-28.

25. Millspaugh J. Dementia pugilistica. US Naval Med Bull 1937; 35 : 297-303.

26. Critchley M. Medical Aspects of Boxing, Particularly from a neurological standpoint. BMJ 1957; 1: 357-362.

27. Miller H. Mental after-effects of head injury. Proc R Soc Med 1966; 59: 257-261.

28. Corsellis J, Bruton C, Freeman-Browne D. The aftermath of boxing. PsycholMed 1973; 3: 270-303.

29. Omalu BI, DeKosky ST, Minster RL, Kamboh MI, Hamilton RL, Wecht CH. Chronic traumatic encephalopathy in a National Football League player. Neurosurg 2005; 57 (1): 128-134.

30. Smith DH, Johnson VE, Stewart W. Chronic neuropathologies of single and repetitive TBI: substrates of dementia? Nat Rev Neurol 2013; 9 (4): 211-221.

31. Omalu B, Hammers JL, Bailes J, Hamilton RL, Kamboh MI, Webster $\mathbf{G}$ et al. Chronic traumatic encephalopathy in an Iraqi war veteran with posttraumatic stress disorder who committed suicide. Neurosurg Focus 2011; 31: E3.

32. McKee AC, Cantu RC, Nowinski CJ, Hedley-Whyte ET Gavett BE, Budson AE et al. Chronic traumatic encephalopathy in athletes: progressive tauopathy after repetitive head injury. J Neuropathol Exp Neurol 2009; 68: 709-735.

33. Hof PR, Knabe R, Bovier P, Bouras C. Neuropathological observations in a case of autism presenting with self-injury behavior. Acta Neuropathol (Berl) 1991; 82: 321-326.

34. Williams DJ, Tannenberg AE. Dementia pugilistica in an alcoholic achondroplastic dwarf. Pathology 1996; 28: 102-104.

35. Jordan BD. The clinical spectrum of sport-related traumatic brain injury. Nat Rev Neurol 2013; 9: 222-230.

36. McKee AC, Stern RA, Nowinski CJ, Stern RA, Daneshvar DH, Alvarez VE et al. The spectrum of disease in chronic traumatic encephalopathy. Brain 2013; 136: 43-64.

37. Mez J, Stern RA, McKee AC. Chronic traumatic encephalopathy: where are we and where are we going? Curr Neurol Neurosci Rep 2013; 13 (12): 407. 